\title{
La formación para la gestión de las redes sociales en los grados de comunicación en España y su adecuación a las competencias demandadas por las organizaciones
}

\section{Education for the management of social networks in communication degrees in Spain and its suitability for skills demanded by organizations}
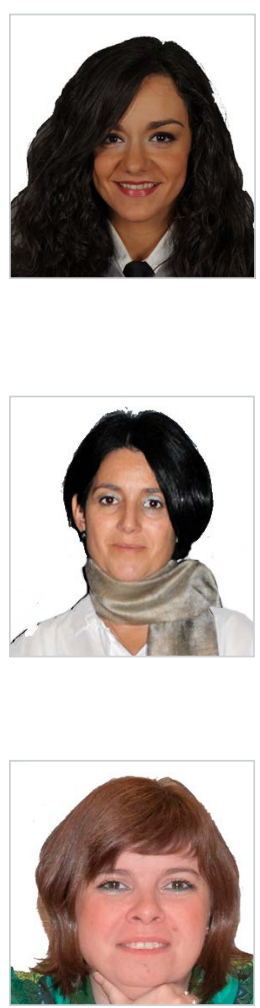

Mariché Navío Navarro. Trabaja en diferentes proyectos del sector de la comunicación online y el marketing digital, compaginando su labor docente e investigadora con la profesional. Es Doctora por la Universidad CEU San Pablo, Máster en Marketing Interactivo \& New Media (IEBS Business School) y licenciada en Periodismo y en Comunicación Audiovisual, además de Título Propio en Nuevas Tecnologías (CEU San Pablo). Como docente, imparte diferentes asignaturas sobre comunicación online y marketing y publicidad digital en el Grado de Comunicación Digital de la Universidad CEU San Pablo. Además, es profesora de varios másteres en esta institución y en la Universitat Oberta de Catalunya. Como profesional, es cofundadora y directora de startups como Dygeat, Communitools.com y Funadtics.

Universidad CEU San Pablo, España

mariajose.navionavarro@ceu.es ORCID: 0000-0002-3511-4505

Laura González-Díez. Profesora titular de la Universidad CEU San Pablo de Madrid. Doctora en Ciencias de la Información por la UCM. Directora del Departamento de Comunicación Audiovisual y Publicidad de la Facultad de Humanidades y Ciencias de la Comunicación (desde 2015). IP del grupo emergente ICOIDI (Investigación en Comunicación a través de la Imagen y el Diseño). Sus investigaciones giran en torno a la Comunicación Digital, al diseño de publicaciones periódicas y a la evolución tipográfica de los diarios y revistas españoles. Ha dirigido 11 tesis doctorales relacionadas con estos temas. De 2015 a 2017 ha sido, además, directora del Servicio de Apoyo a la Investigación de la Universidad CEU San Pablo SAI-CA.

Universidad CEU San Pablo, España

design@ceu.es

ORCID: 0000-0003-1209-8845

Belén Puebla-Martínez. Profesora de la Universidad Rey Juan Carlos. Doctora en Ciencias de la Comunicación por la URJC. Licenciada en Periodismo (2005) y en Comunicación Audiovisual (2007) por la URJC. Es especialista en el estudio de la ficción televisiva española, en el análisis de los medios de comunicación especialmente en prensa y televisión, en el estudio de los métodos analíticos de investigación en comunicación social, en metodologías didácticas aplicadas al aprendizaje del profesorado y en el análisis del uso de las redes sociales como forma de comunicación, entre otras líneas de investigación.

Universidad Rey Juan Carlos, Madrid, España

belen.puebla@urjc.es

ORCID: 0000-0002-1481-4238

Cómo citar este artículo:

Navío Navarro, M.; González-Díez, L. y Puebla-Martínez, B.(2018). La formación para la gestión de las redes sociales en los grados de comunicación en España y su adecuación a las competencias demandadas por las organizaciones. Doxa Comunicación, 26, 127-143. 
Recibido: 27/04/2018 - Aceptado: 29/04/2018 Resumen:

El presente trabajo tiene como objetivo principal realizar un análisis pormenorizado de la oferta formativa sobre la gestión de redes sociales en los grados de comunicación en la universidad española, en el marco del EEES, y comprobar si se adecúa en materia curricular y metodológica a las necesidades del mercado de los distintos perfiles laborales propios del community management. En cuanto a la metodología, se han seleccionado tres técnicas de análisis para la obtención de los objetivos propuestos y la verificación de las hipótesis, de tal manera que proporcionemos al estudio una triangulación metodológica que dé validez a los resultados obtenidos. Estos resultados se han agrupado atendiendo a diferentes criterios, desde la especificidad y carácter de las asignaturas analizadas hasta el curso en el que se imparten. En el análisis, se determina si las materias que incluyen contenidos teóricos o prácticos referidos a la comunicación en redes sociales permiten la adquisición de las competencias específicas necesarias para la óptima empleabilidad de los egresados en profesiones vinculadas a la gestión de la comunicación de marketing en estas plataformas. Todo ello ha permitido obtener como principal conclusión que las universidades españolas no incluyen materias específicas sobre redes sociales de manera transversal en los planes de estudios de sus titulaciones, a lo que se une el hecho de que las universidades que sí ofertan asignaturas específicas sobre redes sociales no adaptan el diseño de estos programas formativos a las necesidades de las empresas en cuanto a los contenidos que incluyen y las competencias que desarrollan en sus alumnos.

\section{Palabras clave:}

Relación universidad-empresa, título universitario, comunicación, community manager, redes sociales, social media
Received: 27/04/2018 - Accepted: 29/04/2018

\section{Abstract:}

The main objective of this research paper is to carry out a detailed analysis of the educational curriculum of social media management in communication degrees at Spanish universities within the framework of the EHEA, as well as to verify whether the curricular content and methodology meet the needs of the labor market linked to different job profiles related to community management. Regarding the research methodology used, three analysis techniques have been selected to achieve the proposed objectives and to verify the hypotheses, and for this reason we have used a methodological triangulation for the study in order to give validity to the results. These results have been grouped according to different criteria, from the specificity and nature of the subjects analyzed to the course in which they are taught. In this analysis, we have attempted to determine whether the subjects that include theoretical or practical contents regarding social media communication allow for the acquisition of the specific skills required for optimum employability of graduates in professions related to the management of marketing communications on these platforms. All of the above has led us to the main conclusion, which is that Spanish universities do not include specific subjects in social networks in a cross-curricular way in their degree programs. Furthermore, we must mention the fact that universities that offer specific subjects related to social networks do not adapt the design of these educational programs to the needs of companies in terms of the content they include and the skills they help their students to develop.

\section{Keywords:}

University-industry collaboration, university degree, communication, community manager, social networks.

\section{Introducción}

La evolución de la World Wide Web a la llamada web 2.0 permitió a los sitios y herramientas alojados en ella dotarse de una capa de interactividad (O’ Reilly, 2005) desconocida hasta el momento (García Estévez, 2012). La socialización de la web en esta segunda etapa llevó a los usuarios a relacionarse entre sí a través de plataformas virtuales, lo que generó redes sociales interconectadas en entornos digitales. Entre los tipos de sites más relevantes de esta web 2.0, los medios sociales (Rodríguez Fernández, 2013), también llamados sitios de redes sociales (SRS) o social media, destacaron por las posibilidades de comunicación multidireccional que permitían a las personas (García Aretio, 2007). Como indica García Estévez (2012: 1) "la web social supuso un antes y un después para el conjunto de la sociedad en todas las esferas de la vida. Desde el plano más personal e íntimo, hasta el profesional o académico”. Las instituciones, inmersas en este proceso de cambio, tuvieron que adaptarse a estas nuevas formas de interrelación entre los individuos para responder a sus necesidades sociales, económicas, tecnológicas y comunicacionales (Huijboom et al., 2009). 
De entre todas, las organizaciones, públicas y privadas, se vieron ante la necesidad de adaptarse a un tipo de público que no había existido hasta entonces (Herreros Laviña, 2013). Esta audiencia buscaba en ellas una relación $P 2 P^{l}$, de igual a igual, basada en una comunicación personal en la que se sintiese escuchada (Redondo y Rojas, 2013). El nuevo mercado, en el que confluían muy distintos tipos de stakeholders $^{2}$, exigía que estas relaciones de intercambio con las marcas se produjesen en los mismos canales en los que ellos se encontraban: los medios sociales y las plataformas 2.0. Las empresas, con más o menos acierto, comenzaron así un proceso de transformación que excedía los límites de la comunicación externa e interna (Levine, Locke, Searls y Weinberger, 2008) para revolucionar desde los propios modelos de negocio (Rodríguez Fernández, 2013) hasta la misma noción del concepto de marketing. En este contexto, las organizaciones toman conciencia de la importancia de formar parte de las comunidades virtuales que hay alrededor de sus marcas y surge la disciplina del community management, encargada de la creación, monitorización, gestión y análisis de las redes sociales conformadas por estos usuarios (Moreno, 2014).

Figuras profesionales como el social media strategist o el community manager, entre otras, nacen para satisfacer la necesidad de las marcas de diseñar su estrategia de comunicación en las redes sociales o de implementar las acciones y gestionar las conversaciones, respectivamente (Mejía, 2014). En una etapa incipiente de esta realidad, la carencia de expertos que cubran estas nuevas vacantes de los departamentos de comunicación provoca que muchas empresas acudan a perfiles como periodistas, publicistas y especialistas en comunicación corporativa o marketing. Otras tantas dejan el manejo de sus medios sociales en manos de becarios, puestos de prácticas generalmente ocupados por estudiantes de Periodismo, Publicidad y Relaciones Públicas, Comunicación Audiovisual, Marketing o Ingeniería Informática. Sin embargo, en poco tiempo la concepción del community management como pieza clave de la comunicación digital de las instituciones provoca la profesionalización del sector y comienzan a demandarse perfiles realmente expertos en la gestión de las redes sociales (Domingo, 2016). Surgen así multitud de cursos, posgrados y másteres para promover la formación profesional de estos nuevos comunicadores (Barrios Rubio y Zambrano Ayala, 2014).

A la par, la universidad, como institución social, comienza a iniciar un proceso de transformación que llega hasta nuestros días. De un lado, la universidad española se ve inmersa en un proceso de adaptación para su convergencia con Europa, en el marco de la creación del Espacio Europeo de Educación Superior (EEES) (Rué, 2007). De otro, la educación superior ha de satisfacer, en el ámbito de la comunicación, nuevas demandas laborales, profesiones para las que se necesita capital humano con una formación especializada (Universia, 2013). Además, la institución universitaria se enfrenta a nuevas exigencias en lo que respecta a sus metodologías de enseñanza, pues las actuales generaciones de estudiantes, como nativos digitales, exigen modelos formativos que los sitúe en el centro de un proceso de aprendizaje más abierto (Adaime, Binder y Piscitelli, 2010) y enfocado al desarrollo de competencias instrumentales (Díaz Barriga, 2008).

1 Las siglas $P 2 P$ hacen referencia al concepto peer-to-peer, red entre iguales o red entre pares, que designa las redes de ordenadores donde no existen clientes y servidores fijos sino un conjunto de nodos que actúan de igual manera, esto es, a la vez como clientes y servidores con respecto al resto de nodos de esa red (Steinmetz y Wehrle, 2005). El uso de este término ha transcendido recientemente al ámbito de la comunicación digital para mencionar la tendencia de los usuarios o consumidores de confiar y dejarse influir más por los mensajes, recomendaciones y opiniones que proceden de sus iguales o pares (dentro de las redes sociales) que por los mensajes que emiten las empresas y marcas (Mootee, 2007).

Usamos aquí el término stakeholder en el sentido amplio propuesto por Ed Freeman en 1983, donde se incluyen a todos aquellos grupos y/o individuos sobre los que una organización depende para supervivencia, además de todos aquellos grupos y/o individuos que puedan afectar o que son afectados por el logro de los objetivos de dicha organización (IESE, 2009). 
La formación para la gestión de las redes sociales en los grados de comunicación en España...

En este sentido, la presente investigación pretende averiguar si la formación universitaria de grado en España se adecúa en materia curricular y metodológica a las necesidades del mercado de los distintos perfiles laborales propios del community management.

\section{Definición del problema de investigación}

El problema que se va a abordar en esta investigación parte de un vacío tanto teórico como empírico a la hora de definir, en primer lugar, cuáles son las competencias y conocimientos que demandan las organizaciones en España para los perfiles laborales de los profesionales especializados en la gestión de la comunicación de marketing a través del canal de los medios sociales en España. En segundo lugar, se pretende analizar la medida en la que la universidad española, para los estudios de grado, es capaz de satisfacer esta demanda de profesionales a través de una oferta formativa que, de un lado, responda a esta necesidad de las organizaciones y, de otro, se adecue a los rasgos del aprendizaje de las generaciones de estudiantes que acceden actualmente a este tipo de estudios: los nativos digitales en el contexto del Espacio Europeo de Educación Superior. En el área teórica, existen numerosas publicaciones que analizan las competencias, funciones, tareas y habilidades de los distintos perfiles profesionales especializados en la comunicación a través de redes sociales. Sin embargo, encontramos un vacío en lo que respecta a la definición específica de estos puestos de trabajo por parte de los profesionales en activo de organizaciones españolas que incluyan estos roles en su organigrama. Las investigaciones existentes al respecto se basan, únicamente, en la figura del community manager en regiones muy específicas como la Comunidad Foral de Navarra y el País Vasco (España) (Elorriaga, 2013), además de en Guatemala (Tobar, 2015). De esta manera, se hace necesario estudiar cuáles son en la práctica profesional las exigencias actuales de las empresas en España a la hora de emplear a este tipo de comunicadores, ya que esto determinará la formación específica que se requiere.

En el área empírica son abundantes las investigaciones que estudian la influencia de las redes sociales en la comunicación de marketing. Este interés se demuestra por la existencia de una vasta literatura que cubre numerosos aspectos: antecedentes y consecuencias de esta comunicación, usos de los medios sociales, persuasión, etc. Numerosas perspectivas que aún dejan un vacío en lo que respecta a la investigación de la formación académica que necesitan estos profesionales.

Por otra parte, contemplamos que en la actualidad, para lograr la satisfacción de la demanda del mercado laboral de este tipo de empleos, multitud de instituciones ofertan cursos de formación en comunicación en redes sociales en los más diversos formatos: másteres y posgrados, cursos monográficos de distintas cargas lectivas, seminarios o certificados, etc. En este heterogéneo ecosistema, encontramos una carencia de estudios publicados que delimiten los contenidos concretos troncales que cualquier formación en gestión de redes sociales tendría que incluir en su currículo.

Más allá, la universidad española actual se encuentra en un proceso de cambio derivado de su integración en el Espacio Europeo de Educación Superior. En los estudios de grado, esta transformación ha propiciado la redefinición de programas y planes de estudio para su adaptación a Europa (Ministerio de Educación, Cultura y Deporte, 2003). Con ella, han surgido nuevas titulaciones universitarias de grado como resultado de dos factores: uno, la conciliación de enseñanzas tradicionales al marco universitario europeo y, dos, la respuesta de la educación superior a las demandas del mercado laboral nacidas en los últimos años. Si bien existen numerosas publicaciones que analizan esta transformación de la universidad española, 
encontramos un vacío en lo tocante a la investigación de su adaptación en el área concreta de los grados de Comunicación que incluyen contenidos para la formación en la gestión de las redes sociales.

Nuestra necesidad de realizar esta investigación parte no solo del vacío teórico y empírico existente sino de una razón pragmática adicional. Esta es la concepción de que este tipo de enseñanzas no han de concebirse únicamente como especializaciones a las que atender desde los programas de másteres y posgrados sino como formaciones transversales en todos los planes de estudio del ámbito de la comunicación, dada la presencia e influencia de los canales de los medios sociales en los ámbitos profesionales a los que dan cobertura los grados en Periodismo, Publicidad y las Relaciones Públicas, Comunicación Audiovisual y, por supuesto, Comunicación Digital y Multimedia.

Esto se debe principalmente a que, primero, este tipo de comunicación a través de los medios sociales todavía no ha alcanzado su madurez (Mejía, 2014) y, segundo, a que la universidad aún está inmersa en la redefinición de su papel en el contexto actual (Ministerio de Educación, Cultura y Deporte, 2010). A pesar de esta carencia de estudios, muchas facultades de comunicación de España ya brindan a sus estudiantes una formación en redes sociales. Estas lo llevan a cabo o bien como asignaturas independientes dedicadas en exclusiva a los medios sociales o bien como contenidos incluidos en asignaturas más amplias. De esta manera, desde una perspectiva práctica, es importante resolver este vacío en la investigación académica para conocer cuál es concretamente la oferta actual de los estudios de grado españoles en materia de redes sociales. Con ello, podremos aproximarnos a la realidad formativa de los estudiantes que se emplearán en el futuro como community managers o social media strategists, entre otros puestos, y determinar cuáles son las carencias del sistema universitario español en este tipo de formación.

Por último, desde la perspectiva teórica, son muchas las investigaciones que tienen como objeto de estudio las redes sociales en la universidad. Este consolidado interés por determinar la influencia de los medios sociales en la institución universitaria ha dado como resultado publicaciones que analizan el fenómeno desde la perspectiva de la creación de entornos virtuales de aprendizaje, la interacción multidireccional de los estudiantes, la percepción de alumnos y docentes sobre las redes sociales de aprendizaje, etc. Sin embargo, no se han encontrado, a excepción de la comunicación de Balandrón y Correyero (2010) sobre el perfil del community manager, investigaciones que conciban la creación y gestión de redes sociales como materia de estudio específica en las titulaciones de comunicación ofertadas en España.

Así las cosas, partimos en esta investigación de la hipótesis de que las Facultades de Comunicación de las universidades españolas poseen una oferta académica que no responde a las demandas que tiene el mercado laboral actual en lo que concierne a los profesionales especializados en la comunicación a través de medios sociales.

\section{El diseño metodológico de la investigación}

Se han seleccionado tres técnicas de análisis para la obtención de los objetivos propuestos y la verificación de la hipótesis, de tal manera que proporcionemos al estudio una triangulación metodológica que dé validez a los resultados obtenidos. El análisis se ha efectuado, cronológicamente, en el siguiente orden:

- Como técnica cuantitativa, el análisis de contenido sobre las publicaciones del ámbito académico y profesional relativas a la definición de las funciones y habilidades de los perfiles profesionales del community manager y el social media 
La formación para la gestión de las redes sociales en los grados de comunicación en España...

strategist. Con este método de investigación podemos conocer, primero, cuáles son las características ideales para estos roles profesionales y, además, estamos en disposición de ofrecer información de valor para la definición de las competencias que analizaremos en el siguiente método de investigación: las entrevistas en profundidad.

- Como técnica cualitativa, entrevistas en profundidad a profesionales en activo del sector de la comunicación en redes sociales, para la definición, según su perspectiva, de las competencias, tareas, funciones, conocimientos y habilidades que estos exigen a los perfiles profesionales encargados de la gestión de los medios sociales. Con esta técnica, podemos determinar las demandas de las empresas y organizaciones españolas en lo que respecta a este tipo de profesionales.

- Como técnica cuantitativa, el análisis de contenido de todas las guías docentes de aquellas asignaturas que, en los grados del ámbito de la comunicación en España, incluyen parcial o totalmente en su currículo contenidos teóricos o prácticos relacionados con la comunicación a través de redes sociales. Con ello pretendemos determinar la oferta académica existente en el curso 2016-2017 en todas las universidades situadas geográficamente en España, sean instituciones públicas o privadas, impartan formación presencial o a distancia para la formación de profesionales en la comunicación en medios sociales, con el objetivo de determinar, posteriormente, el grado de adecuación existente entre esta oferta y las demandas organizacionales analizadas con anterioridad.

Figura 1. Metodología y técnicas de investigación utilizadas.

\begin{tabular}{|l|l|l|l|}
\hline Metodología & \multicolumn{1}{|c|}{ Técnica de investigación } & \multicolumn{1}{|c|}{ Población objeto de estudio } & \multicolumn{1}{|c|}{ Muestra } \\
\hline Cuantitativa & Análisis de contenido & $\begin{array}{l}\text { Publicaciones realizadas entre 2010 y 2017, en español, } \\
\text { relativas a la definición de funciones y habilidades del } \\
\text { community manager y el social media strategist }\end{array}$ & $\begin{array}{l}\text { 85 publicaciones sobre el perfil del community } \\
\text { manar } \\
40 \text { publicaciones sobre el perfil del social media } \\
\text { strategist }\end{array}$ \\
\hline Cualitativa & Entrevistas en profundidad & $\begin{array}{l}\text { Profesionales en activo del sector de la comunicación a } \\
\text { través de medios sociales en España }\end{array}$ & $\begin{array}{l}11 \text { profesionales que desarrollan funciones } \\
\text { propias del social media marketing en 2017 }\end{array}$ \\
\hline Cuantitativa & Análisis de contenido & $\begin{array}{l}\text { Asignaturas que, incluidas en el plan de estudios de los } \\
\text { 170 grados universitarios del ámbito de la comunicación } \\
\text { en España, contienen, para el curso 2016-2017 activida- } \\
\text { des formativas o contenidos teóricos relacionados con la } \\
\text { comunicación en redes sociales }\end{array}$ & 413 asignaturas \\
\hline
\end{tabular}

\section{Fuente: Elaboración propia.}

Para determinar cuáles son las funciones y competencias que la literatura científica y profesional otorga en la actualidad a los profesionales que se encargan de la gestión de la comunicación a través de las redes sociales se optó por diseñar un sistema de categorías dicotómicas, donde se constituyó como variable cada una de las funciones o habilidades que se pueden atribuir a los perfiles profesionales del community manager y del social media strategist, de forma que la categoría determinaba la presencia o ausencia de esa habilidad o función en el texto propio de la unidad de registro.

Para las entrevistas en profundidad partimos de la conjunción de varias informaciones que, provenientes de diferentes fuentes, nos permitieron generar un conjunto de preguntas abiertas a las que daríamos respuesta de manera semiestructurada y con dependencia del avance de cada una de las entrevistas.

En lo que respecta a los entrevistados escogidos, el motivo principal de su elección partió de la consideración de que en esta investigación era necesario contemplar los diversos tipos de organizaciones existentes España, no solamente desde 
la perspectiva de las tipologías de empresa, atendiendo a su tamaño o modelo de negocio, sino también desde el punto de vista de que existen profesionales de las redes sociales que desempeñan su trabajo en instituciones públicas u organizaciones no gubernamentales. De este modo, si bien no hemos seleccionado una muestra representativa del universo, procuramos incluir la mayor variedad de supuestos posibles para evitar el sesgo de los resultados.

Para el análisis de contenido de las guías docentes hemos considerado que ha de estar enfocado principalmente a analizar aquellas de las asignaturas con arreglo al fomento de la adquisición de las competencias que se consideran necesarias para el desempeño de labores profesionales en el ámbito de la comunicación de marketing en redes sociales. Por ello, en lugar de redundar en aspectos formales o estructurales de las guías docentes se decidió tomar como variables las competencias específicas que habrían de adquirirse por los alumnos de cara a mejorar su empleabilidad en el sector y, como categorías, la presencia o ausencia de dichas competencias en las unidades de análisis.

\section{Resultados}

La información obtenida a partir del trabajo de campo realizado se presenta agrupada según diferentes aspectos.

\subsection{Asignaturas de los planes de estudios de los grados del ámbito de la Comunicación que incluyen conte- nidos sobre medios sociales}

Partiendo de los planes de estudio de estos grados, se llevó a cabo el análisis de un total de 475 asignaturas de todo el territorio español en las que se encontraron contenidos teóricos o prácticos, al menos de manera parcial en el currículo de la materia, relacionados con la comunicación en medios sociales. De ellas, 412 tenían las guías docentes disponibles y 16 las tenían accesibles pero pertenecían a grados cuyos planes de estudio no ofrecían todos estos documentos. Por su parte, un total de 47 asignaturas detectadas no tenían las guías docentes disponibles y no se pudieron analizar cuando, posiblemente, por los datos ofrecidos en el portal web de las mismas, estas incluían este tipo de contenidos sobre redes sociales.

\subsection{Asignaturas con contenidos sobre redes sociales según su especificidad}

Una de las variables que más determinan, a nuestro juicio, la adecuación de la oferta formativa de los grados en comunicación en España en materia de redes sociales es la especificidad de las asignaturas que incluyen este tipo de contenidos. Esto, porque si bien son muchas las materias que incluyen unidades didácticas, principalmente teóricas, sobre la influencia de los medios sociales en la comunicación actual de una manera más o menos sucinta, son pocas las asignaturas que se han encontrado cuyo currículo verse de manera íntegra sobre la comunicación a través de las redes sociales.

Son estas últimas, menores en cuantía que las primeras, las que hemos denominado en esta investigación 'asignaturas específicas', las cuales nos permitirán valorar la importancia que las universidades españolas otorgan a este ámbito, ya que la generación en los planes de estudio de los grados en comunicación de materias específicas de este tipo es, sin duda, síntoma de la consideración determinante que tienen los medios sociales como canal de comunicación actual. De esta forma, de las 413 asignaturas encontradas en todo el territorio español donde se incluyen contenidos teóricos o prácticos sobre las redes sociales, solamente se han clasificado 20 como asignaturas específicas, mientras que las 393 restantes no lo son. 
La formación para la gestión de las redes sociales en los grados de comunicación en España...

Son categorizadas como materias específicas aquellas que: a) incluyen en su denominación palabras clave relacionadas con las redes sociales; b) aquellas que, con independencia de su nomenclatura, tengan un porcentaje de contenidos teóricos sobre redes sociales igual o superior al $40 \%$ con respecto al total de contenidos teóricos de la asignatura; o c) aquellas que, con independencia de su nomenclatura, tengan un porcentaje de actividades formativas prácticas sobre redes sociales igual o superior al $40 \%$ con respecto al total de actividades formativas de la asignatura.

Por tanto, teniendo en cuenta que se han analizado 170 planes de estudio y que solamente se han hallado 20 asignaturas específicas sobre redes sociales, lo que supone una proporción del $0,12 \%$, podemos determinar que en la universidad española en la actualidad, no se contempla la formación en redes sociales como necesaria y transversal en los planes de estudio de los grados del ámbito de la comunicación. Por ello, la oferta académica a este respecto no se adecua ni satisface las demandas empresariales para los profesionales del social media.

\subsection{Porcentaje medio de contenidos teóricos sobre medios sociales sobre el total de contenidos de las asigna- turas específicas sobre redes sociales}

De las 20 asignaturas que hemos considerado específicas atendiendo a los criterios expuestos, un total de 10 incluyen contenidos teóricos que versan íntegramente sobre la comunicación en redes sociales. En los 10 casos restantes, la proporción de estos contenidos es menor. A continuación, ofrecemos una tabla con las 10 asignaturas consideradas específicas sobre redes sociales según los criterios mencionados en la metodología pero cuyos contenidos no versan íntegramente sobre este ámbito de estudio:

Figura 2. Asignaturas específicas sobre redes sociales cuyos contenidos no versan íntegramente sobre este ámbito de estudio.

\begin{tabular}{|l|r|}
\hline \multicolumn{1}{|c|}{ Asignatura específica sobre redes sociales } & \% contenidos relacionados con las redes sociales sobre el total \\
\hline Tecnologías digitales aplicadas a la Publicidad & 50,0 \\
\hline Nuevas Tecnologías & 50,0 \\
\hline Introducción al Periodismo Multimedia & 50,0 \\
\hline Nuevas Tendencias en Comunicación Publicitaria & 50,0 \\
\hline Nuevas Tecnologías en Comunicación & 46,7 \\
\hline Nuevas Tecnologías & 42,9 \\
\hline Teoría y Estructura de la Comunicación Digital & 40,0 \\
\hline Nuevas Tecnologías de los Medios Audiovisuales & 40,0 \\
\hline Digital Marketing, Social Media \& Performance & 30,0 \\
\hline Social Media I RP 2.0 & 20,0 \\
\hline
\end{tabular}

Fuente: Elaboración propia.

En estas 10 asignaturas, encontramos que todas son semestrales, de 6 créditos ECTS y, además, ninguna supera el $50 \%$ de sus contenidos teóricos sobre redes sociales. Por ello, la cantidad de horas de estudio dedicadas a asuntos realmente vinculados a la comunicación en medios sociales es insuficiente para la adquisición de las competencias que los futuros profesionales del social media necesitan según las exigencias de las empresas españolas. 
Teniendo en cuenta todas las asignaturas específicas sobre redes sociales en conjunto, encontramos que, de media el porcentaje dedicado a contenidos sobre medios sociales es del $71 \% \mathrm{y}$, sin embargo, en pocos casos como en el Grado en Comunicación Digital de la Universidad CEU San Pablo o en los grados en Comunicación Audiovisual y Publicidad y Relaciones Públicas de la Universidad Camilo José Cela esto resulta suficiente para una formación óptima del alumnado en los asuntos que investigamos.

\subsection{Porcentaje medio de contenidos prácticos sobre medios sociales sobre el total de actividades formativas de las asignaturas específicas sobre redes sociales}

Analizamos ahora, en términos de dedicación, los contenidos prácticos que existen en el conjunto de actividades formativas de las asignaturas específicas. De esta manera, con independencia del número de créditos ECTS, encontramos que el $50 \%$ de estas asignaturas versan íntegramente sobre la comunicación en redes sociales, pero que en el resto de los casos la inclusión de estos contenidos prácticos es menor.

En esta parte de la investigación hemos hallado una gran limitación que nos ha impedido obtener los resultados adecuados. Esta ha venido derivada del uso de las guías docentes de las asignaturas como unidad de análisis. El límite se ha encontrado en el hecho de que en las mayoría de las asignaturas analizadas no se especifican las actividades prácticas que se llevan a cabo en el programa de la asignatura, además de en el hecho de que en algunas de estas materias no se imparten actividades prácticas.

A continuación ofrecemos una tabla con las tres asignaturas cuyo contenido versa íntegramente sobre redes sociales y de las que, de las 20 asignaturas específicas halladas, se incluyen las actividades formativas prácticas en la guía docente:

Figura 3. Porcentaje medio de contenidos prácticos sobre medios sociales sobre el total de actividades formativas de las asignaturas específicas sobre redes sociales.

\begin{tabular}{|c|c|}
\hline Asignatura específica sobre redes sociales & \% actividades prácticas relacionadas con las redes sociales sobre el total \\
\hline Creación y Gestión de Redes Sociales & 100 \\
\hline Gestión de Comunidades Virtuales & 100 \\
\hline Nuevas Tendencias en Comunicación Publicitaria & 57,5 \\
\hline
\end{tabular}

Fuente: Elaboración propia.

\subsection{Asignaturas que incluyen contenidos sobre redes sociales según el curso en que se imparten}

Una de las preguntas de investigación incluidas en el planteamiento de este trabajo plantea que el curso específico en el que se imparten las asignaturas, dentro de un plan de estudios, es un indicador del grado de especialización de dicha asignatura. Esto, porque se considera que cuanto más avanzado sea el curso, mayor conocimiento tendrá el alumno que recibe la materia y, por tanto, más especializada podrá ser la formación impartida. Por tanto, la pregunta aludía a la posibilidad de que, para la optimización de la enseñanza de la comunicación en redes sociales, las asignaturas que incluyen estos contenidos se impartan en los últimos cursos de las titulaciones en las que se encuentren. De las 413 asignaturas que incluyen de manera total o parcial contenidos sobre la comunicación en redes sociales se ha encontrado que la mayoría se imparten en el tercer curso, seguido del segundo y el cuarto, lo que es un indicativo de que los conocimientos adquiridos en ellas podrían ser avanzados. 
La formación para la gestión de las redes sociales en los grados de comunicación en España...

4.6. Adquisición de competencias específicas referidas a conocimientos disciplinares sobre redes sociales en las asignaturas específicas sobre la comunicación en medios sociales

Buena parte de las preguntas de investigación hacen referencia a la adquisición o no, por parte de los alumnos que cursan asignaturas específicas sobre comunicación en redes sociales, de un conjunto de competencias específicas referidas a conocimientos disciplinares sobre redes sociales. Se trata de un grupo de competencias definidas en el Libro blanco para el diseño de las titulaciones universitarias en el marco de la Economía Digital (2015) y que, en el caso de los conocimientos disciplinares, se refieren, principalmente, a la adquisición de conocimientos teóricos sobre la disciplina comunicación en social media.

Cada una de estas competencias específicas son divididas, en la obra mencionada, en una serie de conocimientos teóricos específicos que se han analizado en esta investigación de manera autónoma y diferenciada para poder delimitar de la forma más precisa posible cuál es la oferta real de la universidad española en materia de comunicación en redes sociales a través de este tipo de asignaturas. Así, atendiendo únicamente a las materias que se han clasificado como específicas, obtenemos los siguientes resultados relativos a la adquisición de competencias sobre conocimientos teóricos de la disciplina del social media (CEDG):

Figura 4. Adquisición de competencias CEDG (Asignaturas específicas de RRSS).

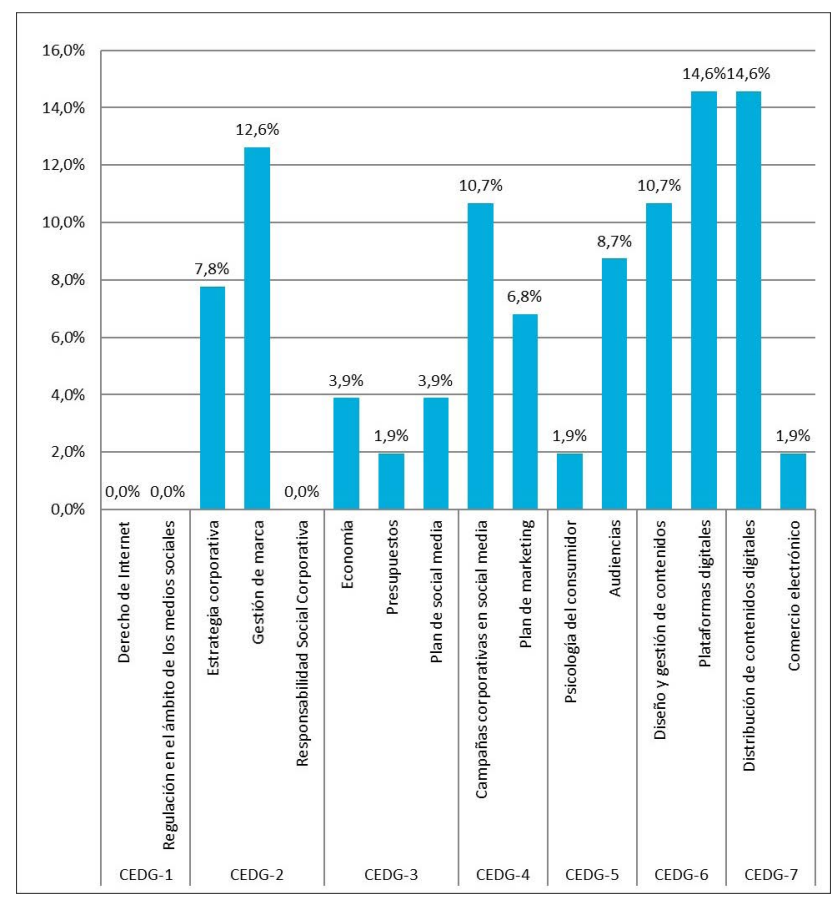

Fuente: Elaboración propia. 
En las asignaturas específicas sobre redes sociales halladas, la adquisición de competencias referidas a conocimientos teóricos sobre la disciplina se fomenta atendiendo a la siguiente distribución:

- La mayoría de estas asignaturas (15 de las 20 analizadas) fomentan el conocimiento teórico de las distintas plataformas digitales que se pueden encontrar en Internet, entre las que encontramos medios sociales, marcadores sociales, agregadores de noticias, wikis, apps, etc.

- En 15 de las 20 materias se promueve la comprensión teórica de los distintos tipos de tácticas o estrategias, canales y prácticas que pueden ser utilizadas para la distribución eficaz de contenidos digitales. Sin embargo, no se han hallado referencias a los resultados económicos derivados de la difusión eficaz de estos contenidos, así como la analítica de resultados.

- En 13 de las materias se impulsa la adquisición de conocimientos teóricos relativos a la gestión de marca, esto es, formación para la difusión correcta de los valores, el posicionamiento y las características que las empresas pretenden asociar a sus marcas hacia el público objetivo (Alonso González, 2015).

- En 11 de las materias se han encontrado contenidos que fomentan el conocimiento teórico de las estrategias y tácticas para la planificación, implementación y difusión de campañas corporativas y de comercialización de productos en social media.

- En 11 de las 20 asignaturas se incluyen unidades temáticas teóricas que incluyen claves y consejos para promover un correcto diseño y gestión de contenidos para captar y mantener la atención de la audiencia. No es un porcentaje alto si tenemos en cuenta que los medios sociales son un canal incluido comúnmente dentro de la disciplina del inbound marketing y de la mano del marketing de contenidos. Los profesionales del social media han de tener, según las opiniones de las empresas españolas, un profundo conocimiento sobre la creación estratégica y gestión de contenidos que solo es apreciable en poco más del $50 \%$ de las materias.

- En nueve asignaturas se instruye teóricamente al alumno para la comprensión de las audiencias de redes sociales, que se estudian, principalmente, desde la perspectiva de los cambios conductuales que estas han sufrido a raíz de la irrupción de las tecnologías digitales propias de la web 2.0. No se han hallado muchos casos en los que estos conocimientos se vinculen a la capacitación del alumno para la predicción de comportamientos y tendencias de la audiencia en relación con un determinado producto. Sí se han encontrado, sin embargo, materias que fomentan el conocimiento sobre la segmentación del público objetivo.

- En ocho asignaturas se instruye para la creación de estrategias corporativas, esto es, conocimientos teóricos para el planteamiento, definición y planificación de estrategias de comunicación relacionadas tanto con el valor de la marca de una empresa como con la promoción de productos o servicios.

- En siete materias se enseña a elaborar un plan de marketing o de comunicación. En las asignaturas más enfocadas al marketing operacional, se alude a la definición de las características propias de las variables del producto, precio, distribución y comunicación. En las más centradas en la comunicación publicitaria, se hace hincapié en la elección de canales para la comunicación del mensaje publicitario a la audiencia objetivo. En la mayoría de ellas se alude a la planificación basada en la definición de objetivos, público objetivo, fases de la estrategia y, solo en algunas ocasiones, medición de resultados. No se trata de un porcentaje de asignaturas óptimo si tenemos en cuenta la demanda empresarial española de que los profesionales propios de los medios sociales sepan desarrollar estratégicamente las acciones de comunicación que llevan a cabo en social media. 
- Las materias que incluyen contenidos relacionados con la creación de planes de social media son solamente cuatro de las 20 asignaturas específicas analizadas. Se trata de un porcentaje muy bajo dada la necesidad, por parte de las empresas, de profesionales propios del sector de las redes sociales que sean capaces de desarrollar estrategias teniendo como canal principal los medios sociales. Por tanto, solamente en cuatro asignaturas se prepara a los alumnos de manera correcta para poder desempeñar las funciones propias del rol profesional del social media strategist, quedando, por tanto, el resto de las asignaturas enfocadas únicamente a poder desarrollar, por parte del alumnado, labores propias de un community manager, quien no define sino implementa la estrategia fijada por el primero.

- También cuatro materias incluyen unidades didácticas teóricas relacionadas con la economía. No hemos hallado en ningún caso contenidos sobre macroeconomía, microeconomía o aspectos financieros; por el contrario, estos temas se centran en entender cómo variables del entorno, organigrama o las debilidades, fortalezas, amenazas y oportunidades de una empresa influyen en la toma de decisiones en comunicación.

- Se han encontrado dos asignaturas donde se tratan teóricamente aspectos relacionados con la psicología del consumidor, mientras que las empresas consideran necesario, para los profesionales del social media, conocer profundamente el comportamiento de su público en redes sociales para poder implementar que logren captarlo, persuadirlo o fidelizarlo.

- Solamente en dos asignaturas hemos encontrado temas que traten la creación de presupuestos, un porcentaje muy bajo que no cubre las necesidades organizacionales al respecto, pues es necesario conocer cómo definir los costes de las acciones en redes sociales para que se ajusten a los recursos financieros y tecnológicos de las empresas.

- También dos asignaturas dedican parte de su temario al comercio electrónico. Consideramos que es necesario aumentar la cantidad de contenidos al respecto ya que las necesidades de las empresas españolas apuntan a que uno de los aspectos para los que utilizan más las redes sociales es para el incremento de las ventas.

- No se ha encontrado en ninguna de las asignaturas específicas sobre redes sociales contenido alguno relacionado con las siguientes temáticas:

- Derecho en Internet y regulación en el ámbito de los medios sociales: Aunque este conocimiento disciplinar es importante para poder desarrollar las funciones propias del social media, la mayoría de los profesionales entrevistados para conocer las demandas empresariales al respecto no consideraron que este conocimiento fuese determinante para poder trabajar como community manager o social media strategist.

- Responsabilidad social corporativa: Ni se incluye en las asignaturas ni los profesionales españoles en activo lo conciben como imprescindible para poder trabajar en medios sociales.

\subsection{Adquisición de competencias específicas referidas a conocimientos prácticos sobre redes sociales en las asignaturas específicas sobre la comunicación en medios sociales}

En lo que respecta a la adquisición de competencias referidas a conocimientos prácticos de la disciplina del social media (CEPG), hemos hallado los siguientes resultados: 
Figura 5. Adquisición de competencias CEPG (Asignaturas específicas de RRSS).

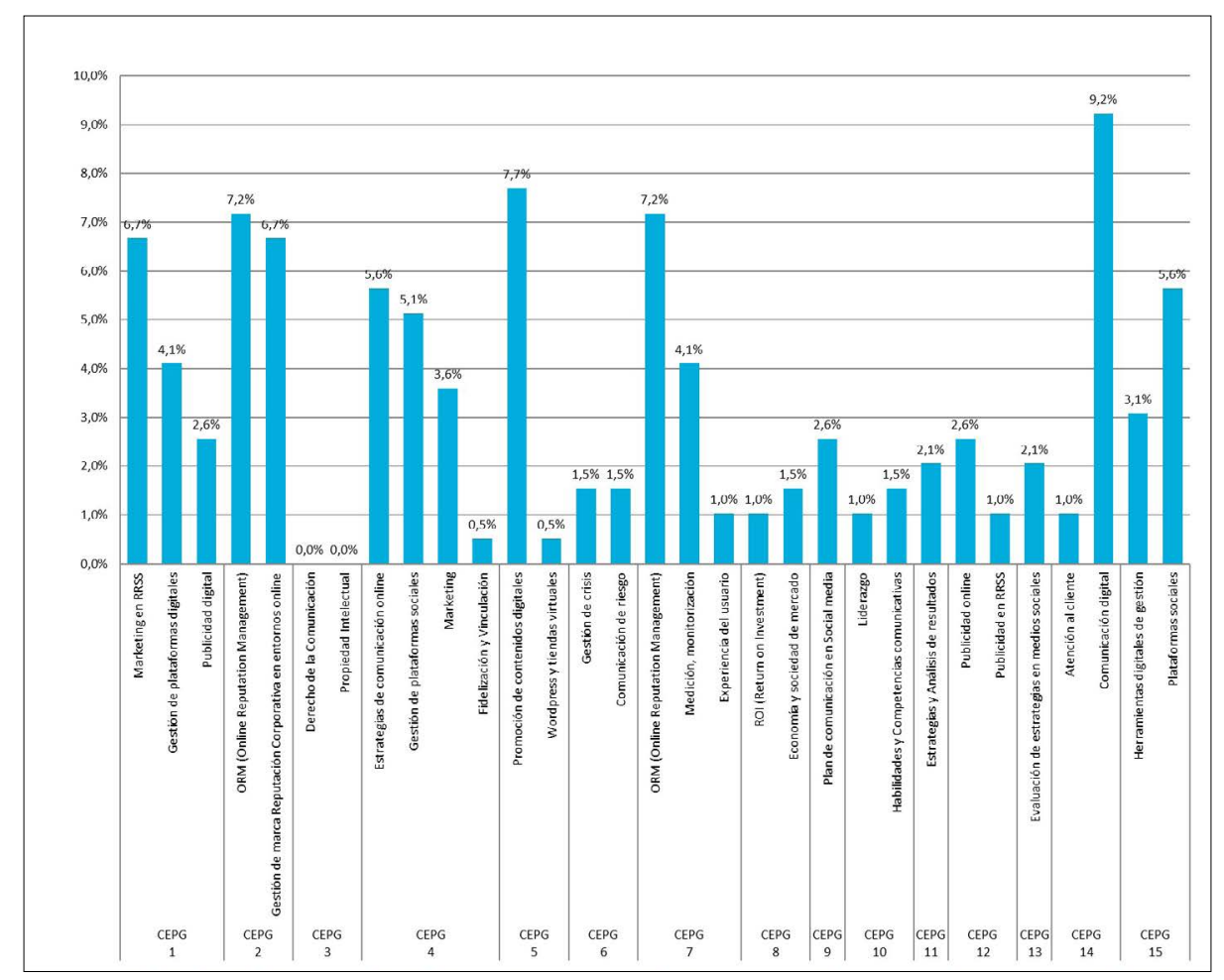

Fuente: Elaboración propia.

4.8. Adquisición de competencias específicas referidas a la gestión de herramientas sobre redes sociales en las asignaturas específicas sobre la comunicación en medios sociales

Los resultados obtenidos son los siguientes:

Figura 6. Contenidos para el aprendizaje de herramientas en asignaturas específicas de RRSS.

\begin{tabular}{|l|c|c|}
\hline \multicolumn{1}{|c|}{ Herramientas } & No & \\
\hline Sitios de RRSS & $26,1 \%$ & 12 \\
\hline Herramientas para la administración y gestión de RRSS (Hootsuite, Tweetdeck...) & 4 \\
\hline $\begin{array}{l}\text { Herramientas para la analítica y monitorización en RRSS y web (Google Analytics, Fb Insights, } \\
\text { Hootsuite...) }\end{array}$ & $3,7 \%$ & $3,5 \%$ \\
\hline Content Management Systems (Wordpress, Joomla, Drupal...) & $6,5 \%$ \\
\hline
\end{tabular}


La formación para la gestión de las redes sociales en los grados de comunicación en España...

\begin{tabular}{|c|c|c|}
\hline CRM o SCRM & 1 & $2,2 \%$ \\
\hline Agregadores de noticias y marcadores sociales (Menéame, Delicious...) & 3 & $6,5 \%$ \\
\hline Wikis & 1 & $2,2 \%$ \\
\hline Plataformas online para crear, almacenar y compartir documentos (Google Drive, Dropbox...) & 2 & $4,3 \%$ \\
\hline Plataformas online para subir y compartir vídeos & 4 & $8,7 \%$ \\
\hline Plataformas online para editar audio & 2 & $4,3 \%$ \\
\hline Plataformas online para editar vídeo & 2 & $4,3 \%$ \\
\hline Photoshop o editor de imágenes similar & 1 & $2,2 \%$ \\
\hline Indesign, QuarkXPress o herramientas de maquetación similares & 1 & $2,2 \%$ \\
\hline Illustrator Freehand o herramientas de diseño gráfico vectorial similares & 0 & $0,0 \%$ \\
\hline Premiere, Final Cut o herramientas de edición de vídeo & 2 & $4,3 \%$ \\
\hline After effects o herramientas similares de motion graphics & 0 & $0,0 \%$ \\
\hline Audition o herramientas similares de edición de sonido & 0 & $0,0 \%$ \\
\hline Dreamweaver o programas de diseño web similares & 0 & $0,0 \%$ \\
\hline Software ofimática (Microsoft Office, Open Office...) & 5 & $10,9 \%$ \\
\hline
\end{tabular}

Fuente: Elaboración propia.

Tras el análisis de contenido de las 413 asignaturas que, para el curso 2016-2017, formaron parte de los planes de estudio de los grados universitarios del ámbito de la comunicación en España, hemos detectado que la oferta académica no forma al alumnado de manera óptima en lo que respecta a las competencias y conocimientos teóricos y prácticos que demandan las empresas y organizaciones para los profesionales que desempeñan funciones relativas a la comunicación de marketing en redes sociales. Mientras que de estas 413 asignaturas, solo 20 pueden considerarse específicas de redes sociales, estas presentan carencias en lo que concierne a la formación para las siguientes competencias exigidas por las empresas: elaboración de presupuestos, planificación estratégica de la comunicación en redes sociales y conocimiento de los públicos y las audiencias. Por su parte, el resto de asignaturas no específicas únicamente forman al alumnado en conocimientos genéricos sobre las redes sociales, referidos a la influencia que estas han ejercido en la comunicación empresarial o en los públicos, por lo que no proporcionan una formación adecuada para el desempeño de las tareas propias de roles profesionales como el social media strategist o el community manager. Por tanto, a la luz de estos resultados, consideramos verificada la hipótesis inicial de que las Facultades de Comunicación de las universidades españolas poseen una oferta académica que no responde a las demandas que tiene el mercado laboral actual en lo que concierne a los profesionales especializados en la comunicación a través de medios sociales. 


\section{Conclusiones generales}

Los resultados obtenidos tras la implementación de los análisis llevados a cabo en los distintos objetos de estudio y a través de las metodologías cuantitativas y cualitativas utilizadas nos permiten determinar las siguientes conclusiones:

En lo que concierne a la oferta académica existente en los grados del ámbito de la comunicación en España hay que señalar que:

1. La actual oferta académica, a nivel de grado, que se produce en las Facultades de Comunicación de las universidades españolas no responde a las demandas que tiene el mercado laboral actual en lo que respecta a la correcta formación de los profesionales especializados en la comunicación a través de los medios sociales, de manera que estas no generan la fuerza de trabajo capaz de cubrir estas necesidades empresariales en la actualidad.

Las asignaturas que, en los grados del ámbito de la comunicación en España, recogen contenidos relacionados con los medios sociales, no tienen un currículo diseñado a partir de la definición de competencias específicas que la ANECA atribuye a los profesionales del social media en su publicación Libro Blanco para el diseño de las titulaciones universitarias en el marco de la economía digital.

2. Las universidades españolas no incluyen materias específicas sobre redes sociales de manera transversal en los planes de estudios de sus titulaciones en comunicación, si bien esta es una exigencia expresada por las empresas españolas.

3. Las universidades que ofertan asignaturas específicas sobre redes sociales no adaptan el diseño de estos programas formativos a las necesidades de las empresas en cuando a los contenidos que incluyen y las competencias que desarrollan en sus alumnos. Así, existen carencias importantes en el conocimiento técnico y profesional de las herramientas, en la planificación estratégica de las comunicaciones en estos canales, en el conocimiento de las audiencias de los medios sociales, en la analítica e interpretación de los resultados, etc. Además, el tiempo dedicado, en términos de créditos ECTS, a estas materias es insuficiente para la adquisición de las competencias que se demandan en el mercado laboral.

4. Las asignaturas no específicas sobre redes sociales, en conjunto, tampoco cubren las necesidades empresariales sobre profesionales de la comunicación en medios sociales y estas centran sus contenidos en una exposición muy genérica de la influencia que estos canales han tenido en los públicos y la comunicación, sin que exista un enfoque que promueva el conocimiento de su uso estratégico.

5. Las asignaturas que incluyen contenidos sobre redes sociales, en conjunto, preparan a los alumnos más para el desempeño de funciones propias del community manager que para la ejecución de tareas estratégicas propias del social media strategist.

6. La localización de las universidades influye en la existencia de formación especializada en redes sociales. Cuanto mayor es la ciudad y la comunidad en términos de población, mayor es, generalmente, la oferta formativa relacionada con la comunicación en redes sociales.

7. Las universidades privadas en España ofrecen una formación más especializada y actualizada en comunicación en redes sociales que las universidades públicas, que sufren un estancamiento mayor en lo que respecta a la incorporación de titulaciones relacionadas con la comunicación y el marketing digital. 
La formación para la gestión de las redes sociales en los grados de comunicación en España...

8. En la universidad española, los únicos estudios que contemplan con mayor frecuencia la formación en comunicación a través de redes sociales como una materia autónoma dentro del plan de estudios son los grados propios del ámbito de la publicidad, relaciones públicas, marketing y comunicación empresarial.

A la luz de estas conclusiones, determinamos que es necesario que los equipos docentes e investigadores de las universidades españolas trabajen en la redefinición de sus planes formativos para la integración de materias específicas transversales que versen sobre la comunicación en redes sociales para todas las titulaciones de grado del ámbito de la comunicación.

Para llevar a cabo el diseño de estos currículos, es necesario un planteamiento que no solo tenga en cuenta las publicaciones académicas al efecto sino que se centre en la cobertura de la necesidades que las empresas españolas presentan en lo que concierne a las funciones, competencias, habilidades y conocimientos que estas exigen a perfiles como el community manager o el social media strategist.

\section{Referencias Bibliográficas}

Adaime, I.; Binder, I. y Piscitelli, A. (comps.) (2010): El proyecto Facebook y la posuniversidad: sistemas operativos sociales y entornos abiertos de aprendizaje. Madrid: Ariel.

Alonso González, M. (2015): “Las redes sociales como canal de comunicación de las marcas de moda españolas. El caso de Zara, Mango y El Corte Inglés”. Index.comunicación, v. 5, n. 1.

http://journals.sfu.ca/indexcomunicacion/index.php/indexcomunicacion/article/view/177 [Consultado el 22/1/2018].

Balandrón, A. J. y Correyero, B. (2010): "La docencia en comunicación en el EEES ante los perfiles profesionales emergentes: el caso de los community managers”. En J. Sierra, y F. Cabezuelo (Coords.). Competencias y perfiles profesionales en los estudios de Ciencias de la Comunicación (pp. 206-219). Madrid: Fragua.

Barrios Rubio, A. y Zambrano Ayala, W.R. (2014): “Formación de comunicadores 'transmedia' para el público de la generación digital”, Index.comunicación v. 4, n. 1. http://journals.sfu.ca/indexcomunicacion/index.php/indexcomunicacion/ article/view/109 [Consultado el 22/1/2018].

Díaz Barriga, F. (2008): “TIC y competencias docentes del S. xxI. Los desafíos de las TIC para el cambio educativo”. En R. Carneiro, T. Díaz y J.C. Toscano, Organización de Estados Iberoamericanos, Metas educativas 2012: La educación que queremos para la generación de los bicentenarios, (pp. 139-155). Madrid: Fundación Santillana.

Domingo, A. (2016): La profesionalización del Community Manager. Recuperado de http://www.iedge.eu/antonio-domingo-la-profesionalizacion-del-community-manager [Consultado el 09/2/2018].

Elorriaga, A. (2013): El Marketing y las Redes Sociales. La figura del Community Manager en las empresas de la Comunidad Autónoma Vasca y la Comunidad Foral de Navarra (Tesis Doctoral). Universidad del País Vasco/Euskal Herriko Unibertsitatea, Vizcaya.

García Aretio, L. (2007): ¿Web 2.0 vs Web 1.0? Recuperado de http://e-spacio.uned.es/fez/eserv/bibliuned:20094/web2vs1. pdf [Consultado el 09/2/2018]. 
García Estévez, N. (2012): Redes Sociales en Internet: Implicaciones y consecuencias de las plataformas 2.0 en la sociedad. Madrid: Editorial Universitas.

Herreros Laviña, P. (2013): El poder es de las personas. Madrid: Léeme Libros.

Huijboom, N. et al. (2009): Public Services 2.0: The Impact of Social Computing on Public Services. Luxemburgo: Institute for Prospective Technological Studies, Office for Oficial Publications of the European Communities. Recuperado de http://ftp. jrc.es/EURdoc/JRC54203.pdf [Consultado el 012/3/2018].

IESE Business School (2009): “La evolución del concepto stakeholders en los escritos de Ed Freeman”. Newsletter no 5-Otro punto de vista. Recuperado de http://www.iese.edu/es/files/La\%20evaluaci\%C3\%B3n\%20del\%20concepto\%20de\%20 stakeholders\%20seg\%C3\%BAn\%20Freeman_tcm5-39688.pdf [Consultado el 06/3/2018].

Levine, R.; Locke, C.; Searls, D. y Weinberger, D. (2008): El manifiesto Cluetrain. Barcelona: Deusto.

McCarthy, E. y Brogowicz, A. (1981): Basic marketing. A managerial approach. Homewood, Ill.: R.D. Irwin.

Mejía, J. C. (2014): La guía del Community Manager. Estrategia, táctica y herramientas. Madrid: Ediciones Anaya Multimedia.

Ministerio de Educación, Cultura y Deporte (2003): La Integración del sistema universitario español en el Espacio Europeo De Enseñanza Superior. Documento-Marco. Recuperado de http://www.eees.es/pdf/Documento-Marco_10_Febrero.pdf [Consultado el 06/3/2018].

Ministerio de Industria, Energía y Turismo (2015): Libro Blanco para el diseño de las titulaciones universitarias en el marco de la Economía Digital. Madrid: Cyan, Proyectos y Producciones Editoriales.

Mootee, I. (2007): Web 2.0 and the Marketing New 4Ps. Recuperado de http://idr.is/wen-20-and-the [Consultado el $09 / 2 / 2018]$.

Moreno, M. (2014): El gran libro del Community Manager. Barcelona: Gestión 2000.

O'Reilly, T. (2005): “What is web 2.0", en: OReilly.com. Disponible en: http://www.oreilly.com/pub/a//web2/archive/whatis-web-20.html [Consultado el 09/2/2018].

Redondo, M. y Rojas, P. (2013): Cómo preparar un plan de Social Media Marketing. Barcelona: Gestión 2000.

Rodríguez Fernández, O. (2013): Curso de Community Manager. Madrid: Anaya Multimedia.

Rué, J. (2007): Enseñar en la Universidad. El EEES como reto para la Educación Superior. Madrid: Narcea Ediciones.

Steinmetz, R. y Wehrle, K. (2005): Peer-to-Peer Systems and Applications. Nueva York: Springer.

Tobar, M. E. (2015): Características del perfil profesional del Community Manager en Guatemala (Trabajo Final de Grado). Universidad Rafael Landívar, Guatemala de la Asunción.

Universia (2013): Ciencias de la Comunicación: cambios que está demandando el mercado laboral para los profesionales de esta área. Recuperado de http://noticias.universia.es/vida-universitaria/noticia/2013/07/17/1036863/ciencias-comunicacion-cambios-esta-demandando-mercado-laboral-profesionales-esta-area.html [Consultado el 06/3/2018]. 